\title{
Optimizing Over-The-Air Virtual Reality Broadcast Transmissions with Low-Latency Feedback
}

\author{
Athul Prasad ${ }^{\dagger}$, Andreas Maeder ${ }^{\ddagger}$, and Mikko A. Uusitalo ${ }^{\dagger}$ \\ ${ }^{\dagger}$ NOKIA Bell Labs, Finland. ${ }^{\ddagger}$ NOKIA Bell Labs, Germany. \\ Email: \{Athul.Prasad, Andreas.Maeder, Mikko.Uusitalo\}@Nokia-Bell-Labs.com
}

\begin{abstract}
Virtual reality is one of the most challenging use cases in $5 \mathrm{G}$ due to the need for simultaneous support for high data rates, low-latency and high-reliability. In this work, we consider a radio resource efficient mechanism for the mass delivery of such content to a multitude of users with D2D augmented 5G broadcast. Based on detailed performance evaluations conducted using $5 \mathrm{G}$ system setting, it is shown that the optimized mechanism can provide significant gains in terms of cell-edge user throughput and resultant spectral requirements for broadcast. The performance gains are obtained due to the ability of the system to optimize the operating point, instead of targeting the worst user which has been the traditional approach for broadcast. The proposed mechanism could be an enabler for mitigating the key challenges currently foreseen for the broadcast of such immersive content.
\end{abstract}

\section{INTRODUCTION}

One of the key difference of the fifth generation (5G) of mobile networks with the previous generations have been the ability to support a wide range of use cases and services, going beyond traditional mobile broadband [1]. While the development of the $5 \mathrm{G}$ network has been motivated by the exponential growth in data rate requirements, the increasing demand has been mainly driven by the development of new applications and services for delivering immersive content for human beings and machines. The standards organization developing the $5 \mathrm{G}$ specifications called third generation partnership project (3GPP) has been initially mainly focusing on unicast or peer-to-peer delivery of extreme mobile broadband data rates, with high-reliability and low-latency [2]. 5G will be supporting a large number of new verticals such as factory automation, automotive, energy / smart grids, health care, media and entertainment (M\&E), connected home, etc. [3], which imposes new constraints on network design as compared to traditional cellular networks.

Virtual reality (VR) as a novel medium for viewing M\&E content has been gaining increasing attention in the recent past, especially due to the significant improvements in video quality. Delivering VR content using wireless networks imposes significant challenges on reliability and latency, but provides significant improvement in the end user experience while consuming such content. The work done in [4] proposes the reliable delivery of such high data rate content ( $\tilde{7} \mathrm{Gbps}$ ) using $\mathrm{WiFi}$, operating in millimeter wave $(\mathrm{mmW})$ frequency bands. Based on advanced compression techniques, the work done in [5] considers the reduction of data rate requirements to 5.2 Gbps. While the earlier works mainly consider the unicast delivery of such content, the challenges for enabling the mass delivery / broadcast of such content to a large number of users using $5 \mathrm{G}$ is presented in [6]. Multi-access edge cloud, which enables the caching and hosting of contents and applications closer to the access network is considered to be a key enabler for optimizing transport and reducing latency [7]. The usage of edge cloud to enable VR content delivery using $\mathrm{mmW}$ bands have been investigated in [8]. Similar to such prior work, the usage of edge cloud to deliver the content to the end user is also used in this work, thereby mitigating possible transport network delays.

Device-to-device (D2D) communication enables user equipment (UE) to directly communicate with each other [9], with limited configuration from the base station (BS) related to the time-frequency resources to be used for communication. D2D broadcast schemes have been investigated in detail both in academia and industry using LTE due to its applicability in enabling public safety / mission-critical communications [10]. The work done in [11] proposes a distributed interferenceaware resource allocation mechanism for D2D broadcast, when a multitude of D2D groups are involved in broadcast. The work done in [12] proposes an incentive framework to enable cooperation between devices using D2D broadcast, to deliver enhanced quality of service. A novel resource allocation mechanism for D2D broadcast based on systematic methods is presented in [13], which ensures higher reliability.

In this work, we consider the combination of downlink and D2D sidelink for optimizing the over-the-air broadcast transmissions in a 5G network, with the assumption of lowlatency and limited feedback. Here the main goal is to reduce the amount of radio resources required to broadcast high data rate VR content, thereby enabling practically viable and costefficient mechanisms for the wireless delivery of such content. The D2D broadcast will augment the broadcast transmissions from the BS, thereby enabling the BS to select an optimal operating point for transmissions. The topic of VR broadcast and the usage of D2D to augment the radio resource efficient delivery of such content has so far received limited attention in literature.

The rest of the paper is structured as follows: Section II gives an overview of the system model used. Section III discusses the optimized D2D augmented broadcast mechanism. Section IV presents the simulation assumptions and system level parameters used for simulations, together with detailed performance results of the proposed scheme. Section V pro- 


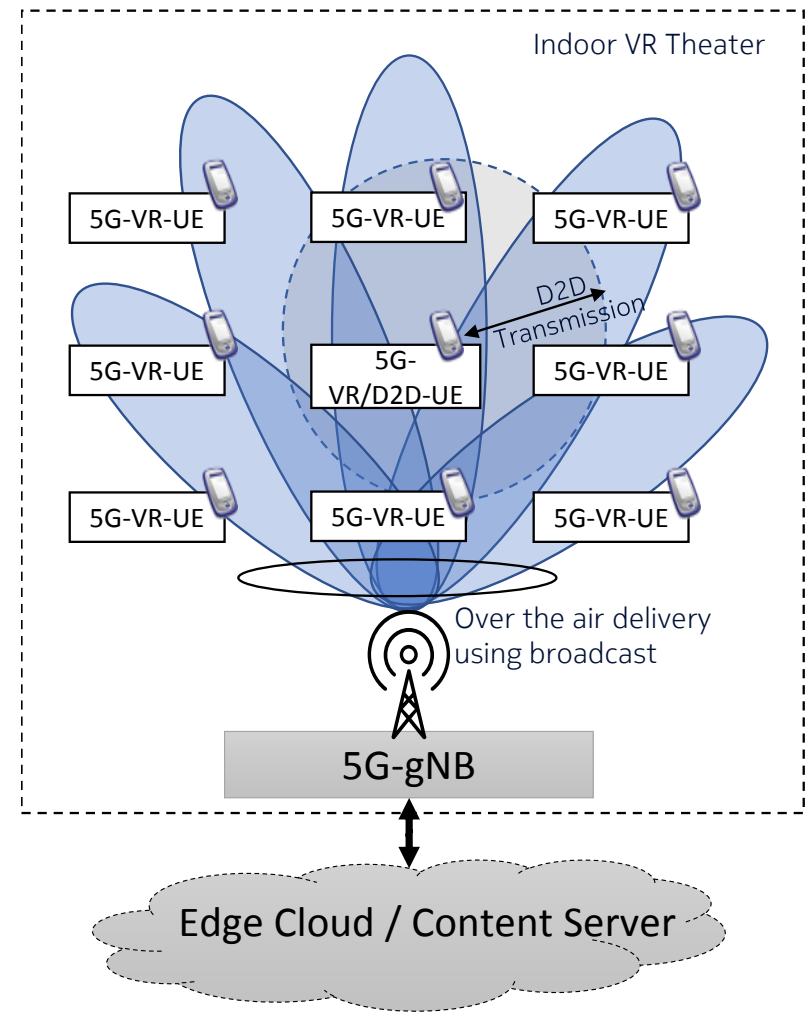

Fig. 1. Overall System Model.

vides a summary of the paper.

\section{SySTEM MODEL}

In this work, we consider a system model similar to the one considered in [6] as shown in Fig. 1, with an indoor broadcast environment with low-mobility assumptions. From content provisioning perspective, we assume the broadcast content to be cached at the edge of the radio access network with minimal transport network delays. The user positioning within the scenario is also considered to be predictable with a fixed set of potential locations where the UE could be located. For performance evaluations, we consider uniform positioning of users in order to estimate the worst-case performance of the proposed enhancements. The base stations within the indoor area are assumed to transmit the broadcast data in a synchronized manner using single frequency network (SFN) concept, with the signal-to-interference and noise (SINR) ratio of the combined SFN transmissions calculated using the model used in [6], [14]. The VR-UEs are also assumed to be capable of D2D transmissions based on the configurations made by the base station. Multiple UEs could be configured to send D2D transmissions in a time synchronized manner using the same radio resources, enabling SFN gains.

Due to the reliability requirement of the VR content, the considered system is assumed to be optimized for delivering broadcast content reliably to all the users, with pure broadcast data transmissions optimized for the worst user. Broadcast is considered here for delivering such common content, instead

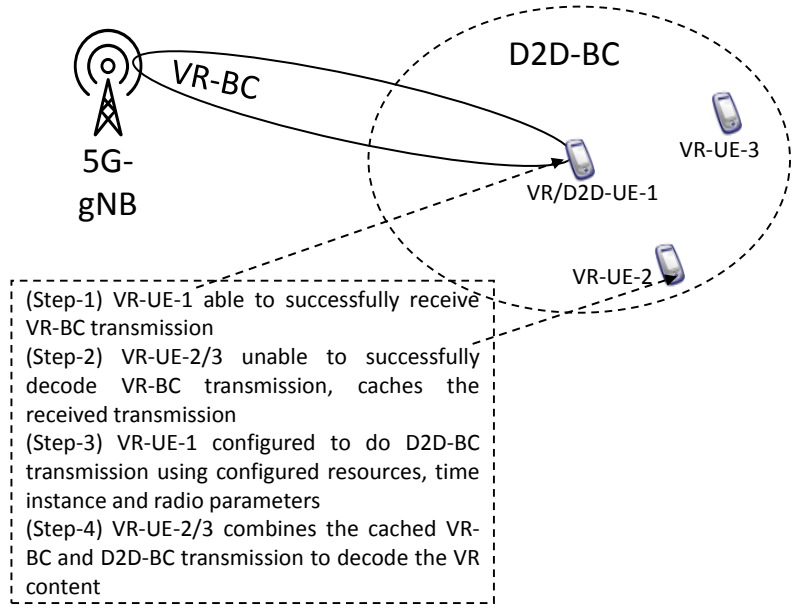

Fig. 2. Configuration steps involved in the proposed mechanism.

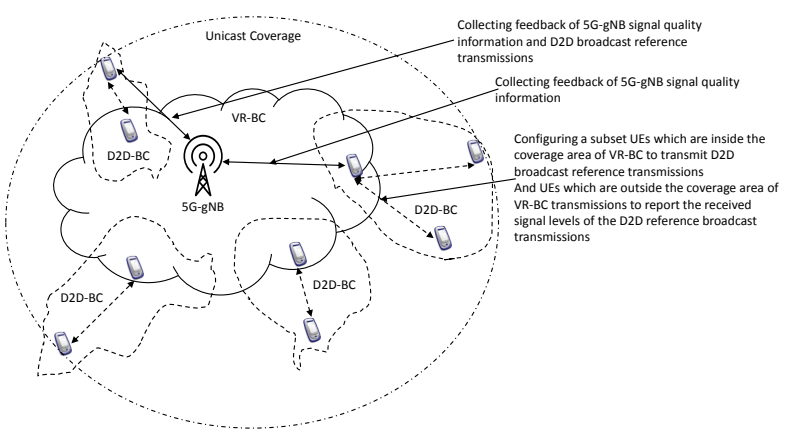

Fig. 3. Scenario setting for optimized broadcast.

of unicast due to the perceived cost advantage in terms of infrastructure and spectrum requirements for delivering such content. While an indoor environment is considered in this work, the proposed mechanisms could be applicable to any generic scenario with limited available spectrum where highquality, high-throughput data needs to be delivered to a large number of users. The VR broadcast transmissions from the base station is termed VR-BC and the assisted D2D broadcast transmissions is termed D2D-BC.

\section{OPTIMIZED BROADCAST WITH LOW-LATENCY FEEDBACK}

A broadcast system is always optimized for the worst user, with the radio parameters such as modulation and coding scheme (MCS), etc., optimized in such a manner that the worst user is also able to receive and decode the transmissions. This imposes severe restrictions on the system if the actual content to be broadcasted is capacity intensive, such as VR. As shown in [6], in a uniformly distributed system, where the users could be placed at any random location within the indoor area, optimizing the system for broadcast in itself is significantly challenging, due to the increased amount of system bandwidth requirement. Adding feedback constraints on such a limited system would put additional constraints 
on the system bandwidth, which cannot be supported in realworld deployments.

In this work, we consider the usage of D2D-BC complementing the VR-BC, so that the system can be optimized for a configured user, rather than the cell-edge / worst user. Here the configured user could be for e.g., mean user in the system, which would ensure that with the combination of D2D and VR-BC, all the users in the system would be covered using the available amount of radio resources. The key enhancement for enabling this is the retransmission of the VR-BC transmission using D2D, possibly with the usage of a configured set of resources. This would also enable the system to minimize the delay, since the VR-BC transmissions can continue even during those time instances where the UE is engaged in D2D-BC transmissions. Since such deployments are assumed to take place within confined indoor areas with a possibly controlled environment, the proposed enhancements can be easily implemented without any standards / 3GPP specification enhancements. The possible steps involved in the method is illustrated in Fig. 2. Here the key assumption is the selection of VR-BC transmission parameters and the preconfiguration of the D2D-BC retransmissions, which ensures coverage and reliability. Here the VR-BC transmissions would be optimized using appropriate parameters targeting a specific set of users, and not the worst user as it is done in traditional $\mathrm{BC}$ networks.

The coverage of the VR/D2D-BC transmissions can be estimated by collecting individual feedback of the VR-UEs, configuring a subset of those UEs which would be potentially inside the coverage area to transmit D2D broadcast reference transmissions and configure subset of those UEs which would be potentially outside the coverage area of VR-BC transmissions to report the received signal levels of the D2D reference broadcast transmissions. The possible configurations for this is as shown in Fig. 3. Here unicast coverage area indicates the coverage footprint of the 5G-gNB while using unicast transmissions with transmission/retransmission parameters adjusted to ensure efficient reception for all users. VR-BC indicates the coverage area for $\mathrm{VR}-\mathrm{BC}$ transmissions, using a particular set of transmission parameters, for e.g., optimized to a target SINR value. Here D2D-BC indicates the coverage area for D2D-BC transmissions, which is estimated using the possible transmission of reference signals. Here the uneven shapes for the coverage areas are used to indicate the possible impacts of shadow-fading and fast-fading which could extend or diminish the coverage in some particular directions. The synchronized VR and D2D-BC transmissions are as shown in Fig. 4, where the D2D-BC retransmissions are configured on either dedicated or reusing VR-BC radio resources while being time synchronized with the VR-BC transmissions.

\section{Simulation Results}

\section{A. Simulation Assumptions}

We consider evaluations based on the parameters used in [6] to better elaborate the possible implementation of the mechanism and the realistic gains that can be achieved. Two

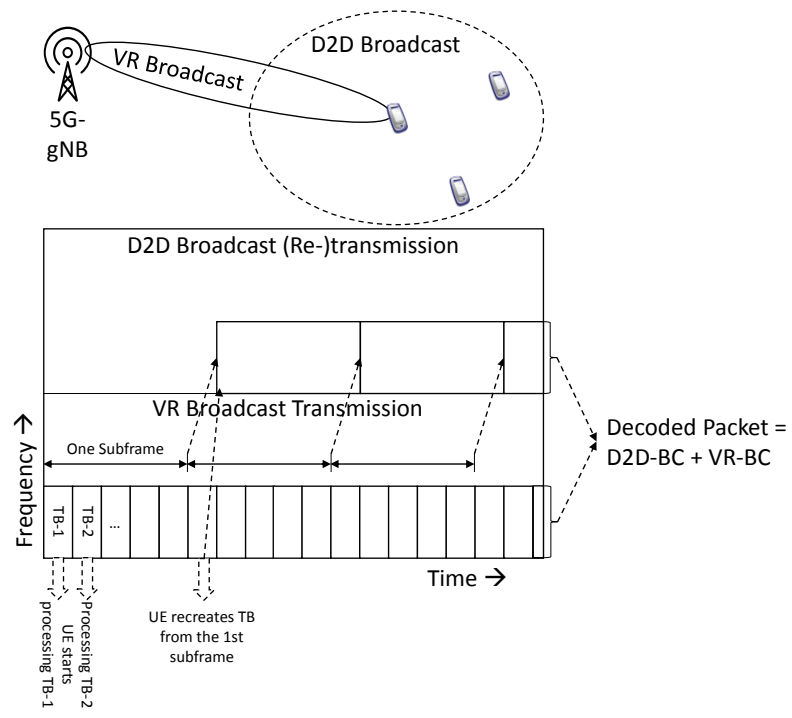

Fig. 4. Synchronized VR and D2D BC.

TABLE I

System LeVel Simulation Parameters

\begin{tabular}{|l|c|}
\hline \multicolumn{2}{|c|}{ Basic Radio Configuration Parameters } \\
\hline Small Cell Deployment & Random, $N_{S C}=4$ \\
\hline Shadowing Standard Deviation & Small Cell: $3 \mathrm{~dB}$ \\
\hline Spectrum Allocation, & $\mathrm{mmW}$ \\
Carrier Frequency & $60 \mathrm{GHz}$ \\
\hline Small Cell Max Tx Power [dBm] & \multicolumn{2}{|c|}{30} \\
\hline Antenna Gain [dB] & Small Cell \\
\hline UE Tx Power [dBm] & 21 \\
\hline \multicolumn{2}{|c|}{ Other Simulation Parameters } \\
\hline Spectral Efficiency, $S_{\text {eff }}$ & 4.0 \\
\hline No. of RBs, $N_{\mathrm{RB}}$ & 5000 \\
\hline PRB size, $R B_{\mathrm{S}}$ & $180 \mathrm{kHz}$ \\
\hline Bandwidth Efficiency, $B_{\text {eff }}$ & 0.65 \\
\hline SINR Efficiency, $S I N R_{\text {eff }}$ & 0.95 \\
\hline User Placement & Random, $N_{U E}=500$ \\
\hline Traffic & Full Buffer \\
\hline
\end{tabular}

reference mechanisms are used as comparisons to evaluate the proposed optimized broadcast mechanism: a) Unicast case is considered as the baseline in order to indicate the worstcase performance in terms of achievable SINR and throughput performance, b) VR-BC case where the entire high data rate content is broadcast without any further optimization. For the optimized VR+D2D-BC case, we set a static minimum SINR for D2D-BC retransmissions to be $12 \mathrm{~dB}$ which could be broadcasted within the network as part of system information. This essentially means that as compared to the VR$\mathrm{BC}$ baseline scenario, using the proposed method, the gNB sets the radio parameters of the $\mathrm{BC}$ transmissions based on a cell-edge or worst user SINR of $12 \mathrm{~dB}$. The VR-UEs having receive SINR greater than $12 \mathrm{~dB}$ would engage in D2D-BC retransmissions. This would enable cell-edge VRUEs (classified here as those having SINR values $<12 \mathrm{~dB}$ ) to cache the VR-BC transmissions and soft-combine it with the D2D-BC transmissions, thereby enabling higher effective SINR values for the cell-edge VR-UEs.

In order to evaluate the technology potential for VR content 


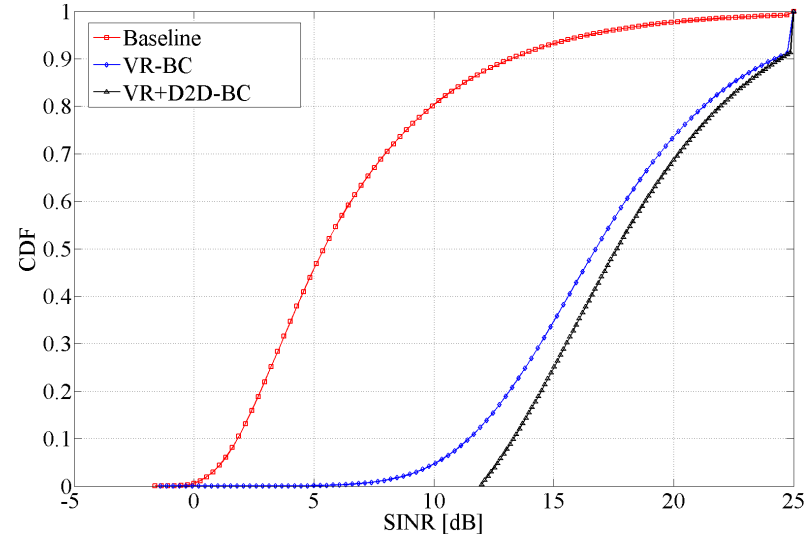

Fig. 5. User SINR distribution.

delivery using broadcast, we consider the uniform deployment of $N_{U E}=600$ VR-UEs using a $5 \mathrm{G}$ system setting. We consider the use of $60 \mathrm{GHz}$ frequency band and a carrier with $1 \mathrm{GHz}$ bandwidth as the baseline for evaluations. The Line-of-Sight (LOS) pathloss model used is based on the one considered in [6], [15], [16]:

$$
L_{5 \mathrm{G}}=92.4+20 \log _{10}(f)+20 \log _{10}(d)
$$

where $\mathrm{f}$ is the carrier frequency in $\mathrm{GHz}$ and $\mathrm{d}[\mathrm{km}]$ is the distance between the VR-UE and LTE small cell. For simplicity, a similar model was also used for the D2D-BC retransmissions.

The detailed parameters used for performance evaluations are as shown in Table I. Only LOS conditions were considered due to the indoor deployment assumptions, with the possibility of planned placement of base stations. $N_{S C}$ small cells are deployed randomly for providing connectivity to the users. Single user traffic was considered for evaluations with the system optimized for delivering traffic to the worst user using broadcast. Unicast throughput results shown as reference in comparison to VR and VR+D2D broadcast considers fullbuffer traffic using round-robin scheduler. Base stations are assumed to have omni-directional antennas, in order to investigate cost-efficient solutions with lower spectral efficiency.

Similar to [6], an indoor setting of $80 \mathrm{~m} \mathrm{x} 60 \mathrm{~m}$ is considered $N_{U E}$ UEs dropped randomly, with a large number of simulation drops used to collect the sufficiently randomized results. A system bandwidth of $1 \mathrm{GHz}$ has been considered as a reference baseline similar to the assumptions in $5 \mathrm{G}$, we also evaluate the spectrum scaling requirements for delivering VR content using broadcast. Here we consider 5.2 Gbps data requirement, based on the evaluations done in [5], thereby using the results in [6] as a baseline for further optimization. The throughput calculations are done based on the modified Shannon formula proposed in [17], with multicast/broadcast specific parameters selected based on the values used in [18].

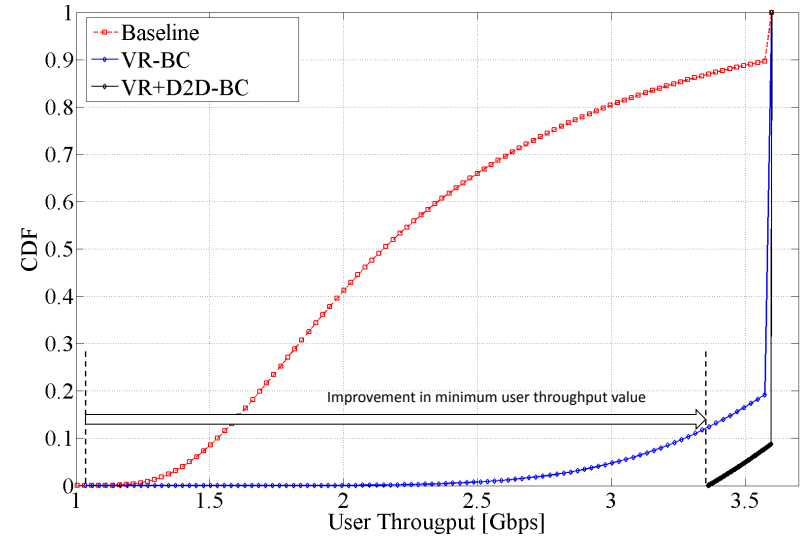

Fig. 6. User throughput distribution.

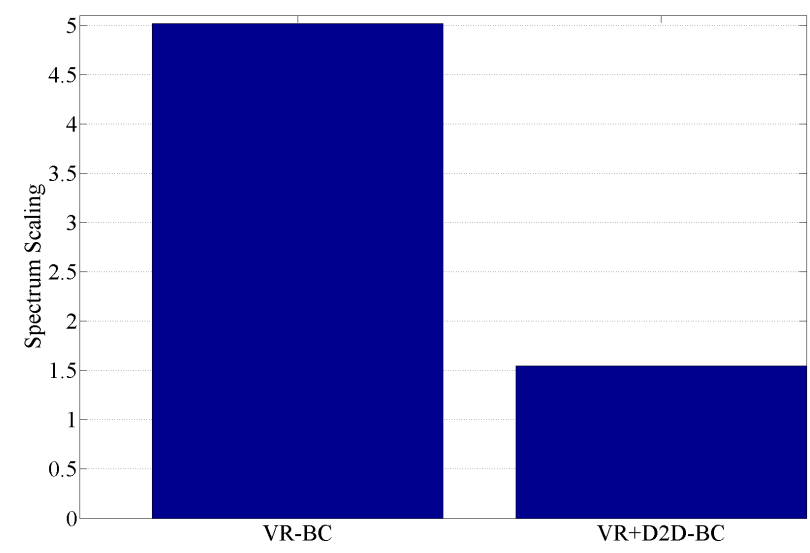

Fig. 7. Spectrum scaling requirements.

\section{B. Simulation Results and Analysis}

In this section, we will discuss the performance results of the optimized broadcast mechanism in comparison to the reference schemes. The SINR distribution is as shown in Fig. 5, with the $12 \mathrm{~dB}$ threshold configured for D2D retransmission. From the figure we can observe that for the optimized broadcast mechanism (VR+D2D-BC) the worst user SINR has improved towards the threshold value. The reason for this is that VR-UEs experience SINR > $12 \mathrm{~dB}$ are statically configured to retransmit the received VR-BC data, using D2D$\mathrm{BC}$ resources. The static threshold assumptions, along with configuration of D2D-BC resources with enable the need for optimized feedback resources for the optimized broadcast scheme.

The user throughput distribution for the various schemes, are as shown in Fig. 5 with the key area of interest for broadcast being worst user throughput. From the figure, we can observe that there is a significant improvement in the cell-edge user throughput for the VR+D2D-BC scheme, as compared to baseline and VR-BC mechanism. The performance of VR$\mathrm{BC}$ mechanism is only marginally better than baseline unicast scheme, indicating that there is no significant improvements 
for cell-edge users in the considered indoor scenario. The results indicate the significant performance gains that could be achieved using the proposed optimized broadcast scheme, as compared to currently available mechanisms.

One of the key performance indicator is the spectrum requirements for delivering fully-immersive VR content using broadcast. Here we consider the spectrum-scaling requirement with $1 \mathrm{GHz} 5 \mathrm{G}$ system bandwidth as the baseline, for delivering VR content using broadcast as compared to the proposed $\mathrm{VR}+\mathrm{D} 2 \mathrm{D}-\mathrm{BC}$ scheme. Thus, the results indicate the amount of additional spectrum required for broadcasting $5.2 \mathrm{Gbps}$ data to the worst user in the scenario using VR-BC in relation to $\mathrm{VR}+\mathrm{D} 2 \mathrm{D}-\mathrm{BC}$, which is shown in Fig. 7. From the figure we can observe that there is significant reductions in the spectrum requirements for $\mathrm{VR}+\mathrm{D} 2 \mathrm{D}-\mathrm{BC}$ due to the ability to deliver higher amount of broadcast data to the cell-edge user experiencing better SINR, as compared to the VR-BC scheme.

The performance evaluations presented in this section indicate the significant gains that could be achieved by optimizing broadcast transmissions with D2D assistance, even with the usage of a static SINR threshold to trigger D2D retransmissions. This enables the base station to optimize the operating point of downlink broadcast transmissions to this configured threshold, with the assumption that sidelink retransmissions will ensure coverage within the system. Here the operating point selection indicates the MCS used by the $5 \mathrm{G}$-gNBs for SFN broadcast transmissions, which is selected based on the worst user in the network for VR-BC scheme, as compared to the static SINR threshold for VR+D2D-BC. For indoor scenarios, such SINR thresholds could be selected based on limited feedback collected from the devices or using limited drive test measurements collected from within the network. While there are several challenges to be addressed in order to enable the mass delivery of immersive content such as VR using $5 \mathrm{G}$, including the need for specifying the support for multicast/broadcast transmissions using 5G/New Radio (NR), the proposed D2D assisted VR broadcast could be considered as a key enabler for delivering such content.

\section{CONCLUSION}

One of the key use cases for mobile networks to support $5 \mathrm{G}$ is the need to deliver high-quality, data-rate intensive traffic such as virtual reality to the end users. In this work, we propose a solution that enables radio resource efficient delivery of such content to a large audience using broadcast transmissions, with limited amount of radio resources. The solution is evaluated using realistic 5G system setting, with key baseline assumptions adapted from LTE broadcast. The performance results indicate that the D2D assisted VR broadcast scheme provides significant gains in terms of spectrum requirements and cell-edge throughput, as compared to a pure broadcast scheme. With the usage of pre-configurations or system information broadcast of the thresholds used, the mechanism could be implemented with limited feedback requirements.

Future work in this area could include further improvements in spectral efficiency using multi-antenna techniques in order to reduce the spectral requirements for the broadcast of high data rate content. Currently in $4 \mathrm{G}$ systems, feedback is not supported for broadcast which could also be a key enabler for improving the performance of the system, for e.g., with the ability to dynamically select the operating point in the D2D assisted VR broadcast scenario.

\section{ACKNOWLEDGMENT}

This work was supported in part by the European Commission under the 5G-PPP project 5G-Xcast (H2020-ICT-20162 call, grant number 761498). The views expressed in this contribution are those of the authors and do not necessarily represent the project.

\section{REFERENCES}

[1] A. Osseiran, F. Boccardi, V. Braun et al., "Scenarios for 5G Mobile and Wireless Communications: The Vision of the METIS Project," IEEE Communications Magazine, vol. 52, no. 5, pp. 26-35, 2014.

[2] S.-Y. Lien, S.-L. Shieh, Y. Huang et al., "5G New Radio: Waveform, Frame Structure, Multiple Access, and Initial Access," IEEE Communications Magazine, vol. 55, no. 6, pp. 64-71, 2017

[3] NGMN Alliance, "5G White Paper," Feb. 2015.

[4] O. Abari, D. Bharadia, A. Duffield, and D. Katabi, "Cutting the Cord in Virtual Reality," in Proceedings of the 15th ACM Workshop on Hot Topics in Networks. ACM, 2016, pp. 162-168.

[5] E. Bastug, M. Bennis, M. Médard, and M. Debbah, "Toward Interconnected Virtual Reality: Opportunities, Challenges, and Enablers," IEEE Communications Magazine, vol. 55, no. 6, pp. 110-117, 2017.

[6] A. Prasad, M. A. Uusitalo, D. Navratil, and M. Saily, "Challenges for Enabling Virtual Reality Broadcast Using 5G Small Cell Network," in IEEE WCNC Workshops (WCNCW), 2018, pp. 1-6.

[7] T. Taleb, K. Samdanis, B. Mada, H. Flinck, S. Dutta, and D. Sabella, "On multi-access edge computing: A survey of the emerging 5G network edge cloud architecture and orchestration," IEEE Communications Surveys \& Tutorials, vol. 19, no. 3, pp. 1657-1681, 2017.

[8] M. S. Elbamby, C. Perfecto, M. Bennis, and K. Doppler, "Edge Computing Meets Millimeter-wave Enabled VR: Paving the Way to Cutting the Cord," arXiv preprint arXiv:1801.07614, 2018.

[9] A. Prasad, A. Kunz, G. Velev, K. Samdanis, and J. Song, "Energyefficient D2D discovery for proximity services in 3GPP LTE-advanced networks: ProSe discovery mechanisms," IEEE vehicular technology magazine, vol. 9, no. 4, pp. 40-50, 2014.

[10] Z. Wu, V. D. Park, and J. Li, "Enabling device to device broadcast for LTE cellular networks," IEEE Journal on Selected Areas in Communications, vol. 34, no. 1, pp. 58-70, 2016.

[11] C.-W. Yeh, M.-J. Shih, G.-Y. Lin, and H.-Y. Wei, "LTE-D broadcast with distributed interference-aware D2D resource allocation," in Seventh International Conference on Ubiquitous and Future Networks (ICUFN), 2015, pp. 165-170.

[12] J. Li, R. Bhattacharyya, S. Paul, S. Shakkottai, and V. Subramanian, "Incentivizing sharing in realtime D2D streaming networks: A mean field game perspective," IEEE/ACM Transactions on Networking, vol. 25, no. 1, pp. 3-17, 2017.

[13] Y. Liu, D. Li, H. Guo, M. Baker, and F.-C. Cheng, "Resource allocation for device-to-device broadcast communication in cellular networks," in Personal, Indoor, and Mobile Radio Communications (PIMRC), 2015 IEEE 26th Annual International Symposium on, 2015, pp. 1178-1183.

[14] L. Rong, O. B. Haddada, and S.-E. Elayoubi, "Analytical analysis of the coverage of a MBSFN OFDMA network," in IEEE Global Telecommunications Conference (GLOBECOM), 2008, pp. 1-5.

[15] M. Cudak et al., "Moving Towards mmWave-based Beyond-4G (B-4G) Technology," in IEEE VTC-Spring, 2013, pp. 1-5.

[16] A. Prasad, F. S. Moya, M. Ericson, R. Fantini, and O. Bulakci, "Enabling RAN Moderation and Traffic Steering in 5G Radio Access Networks," in IEEE VTC-Fall, 2016, pp. 1-5.

[17] P. Mogensen, W. Na, I. Z. Kovács et al., "LTE capacity compared to the Shannon bound," in IEEE 65th Vehicular Technology Conference, 2007, pp. $1234-1238$. 
[18] A. Prasad, A. Maeder, K. Samdanis et al., "Enabling Group Communication for Public Safety in LTE-Advanced Networks," Journal of Network and Computer Applications, vol. 62, pp. 41-52, 2016. 